\title{
SOBRE LA EXISTENCIA DEL PRODUCTO DE MEDIDAS VALORADAS EN ESPACIOS LOCALMENTE CONVEXOS: UNA CONDICION NECESARIA
}

FIDEL JOSÉ FERNÁNDEZ Y FERNÁNDEZ-ARROYO

Abstract

A necessary condition is given for the existence of the tensor product of certain measures valued in locally convex spaces.

1. Introducción y Preliminares. En un reciente trabajo ([7], [8]) abtuvimos condiciones suficientes para garantizar, en el contexto de los espacios localmente convexos, la existencia y la representación integral del producto de dos medidas $\alpha$ y $\beta$ con valores en un espacio de operadores. El objetivo del presente artículo es probar que, al menos en determinadas situaciones, alguna de estas condiciones es también necesaria, a semejanza de lo que ocurría en el caso bilineal considerado por S.A. Sivasankara [14] y R. Chivukula y Sastry [11].

En lo sucesivo, $X, Y$ y $S$ serán e.l.c. separados, siendo $Y$ y $S$ completos; denotaremos por $P, Q$ y $S$ sendas familias generantes y dirigidas de seminormas continuas de estos espacios. Consideraremos, en el espacio vectorial $L(X, Y)$ (resp. $L(Y, S), L(X, S)$ ) de las aplicaciones lineales y continuas de $X$ en $Y$ (resp. de $Y$ en $S$, de $X$ en $S$ ), la topología localmente convexa de la convergencia puntual. $(\Omega, \mathscr{A})$ y $(E, \xi)$ serán espacios medibles; $\alpha: \mathcal{A} \longrightarrow L(X, Y)$ y $\beta: \xi \longrightarrow$ $L(Y, S)$, medidas contablemente aditivas.

Para cada par de seminormas $q \in Q$ y $p \in \mathcal{P}$, llamaremos semivariación de $\alpha$ asociada a $q$ y $p$ a la aplicación

$$
\|\alpha\|_{Q, p}: A \longrightarrow \mathbb{R}^{+} \cup\{+\infty\}
$$

definida por

$$
\|\alpha\|_{q, p}(A)=\sup \left\{q\left(\sum_{i=1}^{n} \alpha\left(C_{i}\right)\left(x_{i}\right)\right)\right\} \quad(A \in A)
$$


donde el supremo se toma sobre todas las particiones medibles finitas $\left\{C_{1}, \ldots, C_{n}\right\}$ de $A$ y todas las colecciones finitas $\left\{x_{1}, \ldots, x_{n}\right\}$ de elementos de $X$ tales que $p\left(x_{i}\right) \leq 1$, para $i=1, \ldots, n$.

Análogamente se define la semivariación de $\beta$ asociada a cada par de seminormas $s \in S$ y $q \in Q$.

Decimos que la medida $\alpha$ (resp. $\beta$ ) es de semivariación acotada si, para cada seminorma $q \in Q$ (resp.s $\in S$ ), existe una seminorma $p \in P$ (resp. $q \in Q$ ) tal que $\|\alpha\|_{q, p}(\Omega)<+\infty$ (resp. $\|\beta\|_{\varepsilon, q}(E)<+\infty$ ).

En adelante supondremos que $\alpha$ y $\beta$ son de semivariación acotada.

Diremos que la medida $\alpha$ es continua si, para cada seminorma $q \in Q$, existe una serninorma $p \in P$ tal que la semivariación $\|\alpha\|_{q, p}$ es continua; es decir, se verifica que, si $\left(A_{n}\right)_{n \in N} \subset \mathscr{A}$ es una sucesión disjunta, entonces la sucesión $\left(\|\alpha\|_{q, p}\left(\cup_{i \geq n} A_{i}\right)\right)_{n \in N}$ converge a cero. (Nótese que, por ser $\alpha$ de semivariación acotada, podemos suponer que $\left.\|\alpha\|_{Q, p}(\Omega)<+\infty\right)$.

Decimos que $\alpha$ verifica la ${ }^{* \prime}$-condición si, para cada $q \in Q$, existen una seminorma $p \in P$, y una medida positiva finita y contablemente aditiva $\nu_{q . p}$ : $A \longrightarrow \mathbf{R}^{+}$, tales que $\|\alpha\|_{q, p} \ll \nu_{q, p} \quad\left(\|\alpha\|_{q, p}\right.$ es absolutamente continua con respecto a $\nu_{q, p}$; e.d., dado $\varepsilon>0$, existe $\delta>0$ tal que, si $A \in A$ y $\nu_{q, p}(A)<\delta$, entonces $\left.\|\alpha\|_{Q, p}(A)<\varepsilon\right)$; puede demostrarse que, en este caso, $\|\alpha\|_{q, p}(\Omega)<+\infty$ (ver $[\mathbf{2}],[\mathbf{1 5}])$.

Diremos que la medida $\alpha: A \rightarrow L(X, Y)$ está acotada en el sentido de Mackey (o es Mackey-acotada) si existe una aplicación $\lambda: \mathscr{A} \longrightarrow \mathbf{R}^{+}$veriffcando:

1) $\lambda$ está acotada.

2) Si $\left(A_{n}\right)_{n \in N} \subset A$ es una sucesión disjunta, entonces la sucesión $\left(\lambda\left(U_{i \geq n} A_{i}\right)\right)_{n \in N}$ converge a cero.

3) Para cada seminorma $q \in Q$, existen $p \in \rho$ y $M_{q}>0$ tales que $q_{x}(\alpha(A)) \leq M_{q} p(x) \lambda(A)$, cualesquiera que sean $x p \in X$ y $A \in A$.

Si se verifica además la condición:

3') Para cada seminorma $q \in Q$, existen $p \in P$ y $M_{q}>0$ tales que $\|\alpha\|_{q, p}(A) \leq M_{q} \lambda(A)$, para todo $A \in A$, entonces $\alpha$ se llama Mackey ${ }^{*}$-acotada.

Nótese que de 3') se deduce 3 ) (ver [7]). Además; se comprueba fácilmente que, si $\alpha$ es Mackey* -acotada, entonces $\alpha$ ha de ser continua. Por otra parte, se prueba en [7] que, si $Y$ es metrizable y $\alpha$ es continua, entonces $\alpha$ es Mackey* -acotada (y, por tanto, Mackey-acotada). (En [7] se pone un ejemplo de una medida que es Mackey*-acotada sin que $Y$ sea metrizable.)

De manera análoga se definen las correspondientes condiciones relativas a la medida $\beta$.

Observación. Si la medida $\beta: \xi \longrightarrow L(Y, S)$ es continua y Mackey-acotada (en particular, si es Mackey* -acotada), entonces verifica la ${ }^{* \prime}$-condición.

En efecto, ya que $\beta$ es Mackey-acotada, existe un conjunto acotado y absolutamente convexo $B \subset L(Y, S)$ tal que $\beta(\xi) \subset L_{B}$ y $\beta: \xi \longrightarrow L_{B}$ es una medida contablemente aditiva (y, por tanto, acotada) (ver\{ 7 , Teorema II.3)\}. 
(Siguiendo la notación de Grothendieck, $L_{B}$ denota el subespacio vectorial generado por $B$, en el que consideraremos la topología definida por el funcional de Minkowski, $p_{B}$.)

Por consiguiente, existe una medida positiva finita contablemente aditiva $\mu: \xi \longrightarrow \mathbb{R}^{+}$tal que $p_{B} \circ \beta \ll \mu$ (ver $\left.[5]\right)$.

Se sigue que, si $\mu(G)=0(G \in \xi)$, entonces $\beta(B)=0$, para todo subconjunto medible $B$ de $G$; y, por tanto, $\|\beta\|_{s, q}(G)=0$, para cada par de seminormas $s \in \mathcal{S}$ y $q \in Q$.

Se deduce de lo anterior que, si la semivariación $\|\beta\|_{\varepsilon, q}$ es continua, entonces $\|\beta\|_{\text {s, } q} \ll \mu$.

2. Resultados. Supongamos ahora que $Y$ es normado, con norma $q$; las medidas $\alpha$ y $\beta$ son Mackey-acatadas, y $\beta$ verifica la ${ }^{*}$ - condición.

Según probamos en [7], [8], en estas condiciones la medida producto $\alpha \otimes \beta$ existe y está dada por

$$
(\alpha \otimes \beta)(G)=\int_{E} \alpha(G .) d \beta \quad(G \in A \otimes \xi),
$$

donde la integral se toma en el sentido de [7] (ver también [9]). (Para cada $s \in E$, denotamos por $G$, al conjunto $\{t \in \Omega /(t, s) \in G\}$, que como sabernos es medible).

Por otra parte, sabemos (ver [5]) que para cada $x \in X$ existe una medida positiva finita contablemente aditiva $\mu_{x}: A \longrightarrow \mathbb{R}^{+}$tal que $q_{x} \circ \alpha \ll \mu_{x}$.

Además, por ser $\beta$ Mackey-acotada, existen un conjunto acotado y absolutamente convexo $B \subset L(Y, S)$ y una medida positiva finita contablemente aditiva $\nu: \xi \rightarrow R^{+}$, tales que $\beta(\xi) \subset L_{B}$ y $p_{B} \cdot \beta \ll \nu$.

Proposición. Para cada $s \in S$ y $x \in X, s_{z}(\alpha \otimes \beta) \ll \mu_{x} \otimes \nu$ es decir,

$$
\lim _{\substack{\left(\mu_{x} \otimes \nu\right)(G) \rightarrow 0 \\(G \in A \otimes G)}} s_{z}((\alpha \otimes \beta)(G))=0
$$

Demostración: Si $0=\left(\mu_{z} \otimes \nu\right)(G)=\int_{E} \mu_{x}(G) d \nu \quad(G \in A \otimes \xi)$, entonces existe $B \in \xi$ tal que $\nu(B)=0$ y $\mu_{x}\left(G_{s}\right)=0$, para todo $s \in E-B ; \mathrm{y}$, por tanto, $\|\beta\|_{s, q}(B)=0$, y $g_{x}\left(\alpha\left(G_{s}\right)\right)=0$, si $s \in E-B$. Se sigute que $s_{x}((\alpha \otimes \beta)(G))=s\left(\int_{E-B} \alpha(G).(x) d \beta\right)=0$.

Y ya que la medida producto $\alpha \otimes \beta$ es contablemente aditiva, resulta de to anterior que $s_{x}(\alpha \otimes \beta) \ll \mu_{z} \otimes \nu$.

Definición. Supongamos que $Y$ es normado (con norma $q$ ), la medida $\beta: \xi \longrightarrow L(Y, S)$ es Mackey-acotada, y $\alpha: \mathcal{A} \longrightarrow L(X, Y)$ es una medida contablemente aditiva tal que la medida producto $\alpha \otimes \beta$ existe.

Según hemos visto, existen un conjunto acotado y absolutamente convexo $B \subset L(Y, S)$; y medidas positivas finitas contablemente aditivas $\nu: \xi \longrightarrow \mathbf{R}^{+}$ 
y $\mu_{z}: A \longrightarrow R^{+}$(para cada $x \in X$ ), tales que $\beta(\xi) \subset L_{B}, p_{B} \cdot \beta \ll \nu$, y $q_{x} \cdot \alpha \ll \mu_{x}(x \in X)$.

Decimos que la medida producto $\alpha \otimes \beta$ hereda la propiedad de dominación si $s_{x}(\alpha \otimes \beta) \ll \mu_{\mathrm{z}} \otimes \nu$ para cada $s \in \mathcal{S}$ y $x \in X$ (y para cada $\mu_{\mathrm{z}}, B$ y $\nu$ verificando las condiciones anteriores). Según hemos visto en la Proposición, $\alpha \otimes \beta$ hereda la propiedad de dominación si las medidas $\alpha$ y $\beta$ verifican las hipótesis del Teorema II.3 de [7].

Definición. Supongamos que $Y$ es normado. Siguiendo a Rao Chivukula y Sastry [11] y S.A. Sivasankara [14], diremos que el espacio $Y$ tiene la propiedad $P$ si el conjunto $B=\{y \in Y / q(y) \leq 1\}$ está contenido en la imagen de una medida vectorial (es decir, existen un espacio medible $(\Omega, A)$ y una medida contablemente aditiva $\alpha: A \longrightarrow L(R, Y) \simeq Y$ tales que $B \subset\{\alpha(A)(1) / A \in$ $A\} \simeq \alpha(A))$. (En $[11]$ y [14] se dan ejemplos de espacios normados que tienen la propiedad $P$.)

Teorema. Supongamos que: la medida $\beta$ es Mackey-acotada; el espacio $Y$ es normado con norma $q$ (por ser $Y$ normado, $\beta$ verifica la $u$-condición, ver (7) ), y tiene la propiedad $P$; $y$, para todo espacio medible $(\Omega, A)$ y toda medida contablemente aditiva $\alpha: A \longrightarrow L(R, Y) \simeq Y$, la medida producto $\alpha \otimes \beta$ existe y además hereda la propiedad de dominación.

Entonces, la medida $\beta$ verifica la ${ }^{*}$-condición.

Demostración: Ya que $Y$ tiene la propiedad $P$, existen un espacio medible $(\Omega, A)$ y una medida contablemente aditiva $\alpha: A \longrightarrow L(R, Y)$ tales que $B=$ $\{y \in Y / q(y) \leq 1\} \subset \alpha(A)(\{1\})=\{\alpha(A)(1) / A \in A\}$.

Por ser $\beta$ Mackey-acotada, existen un conjunto $B \subset L(Y, S)$, acotado, convexo y equilibrado; y una medida positiva finita contablemente aditiva $\nu: \xi \longrightarrow \mathrm{R}^{+}$, tales que $\beta(\xi) \subset L_{B}$ y $p_{B} \cdot \beta \ll \nu$.

Por otra parte, puesto que $\alpha$ es contablemente aditiva, existe una medida positiva finita contablemente aditiva $\mu: A \rightarrow \mathbb{R}^{+}$tal que

$q_{1} \cdot \alpha \ll \mu$.

Pongamos $K=\mu(\Omega)+1$.

Sea $s \in S$. Veamos que $\|\beta\|_{s, q} \ll \nu$.

Por hipótesis, la medida producto $\alpha \otimes \beta$ existe y hereda la propiedad de dominación. Por tanto, dado $\varepsilon>0$ existe $\delta>0$ tal que, si $(\mu \otimes \nu)(G)<\delta(G \in$ $A \otimes \xi\}$, entonces $s_{1}(\{\alpha \otimes \beta)(G))<\varepsilon$.

Se tiene que, si $B \in \xi$ verifica que $\nu(B)<\frac{\delta}{K}$, entonces, para cada partición medible y finita $\left\{B_{1}, \ldots, B_{n}\right\}$ de $B, y$ para cada colección $\left\{A_{1}, \ldots, A_{n}\right\} \subset A$,

$$
\begin{aligned}
(\mu \otimes \nu)\left(\cup_{i=1}^{n} A_{i} \times B_{i}\right) & =\sum_{i=1}^{n}(\mu \otimes \nu)\left(A_{i} \otimes B_{i}\right)=\sum_{i=1}^{n} \mu\left(A_{i}\right) \cdot \nu\left(B_{i}\right) \leq \\
& \leq K \cdot\left(\sum_{i=1}^{n} \nu\left(B_{i}\right)\right)=K \cdot \nu(B)<\delta
\end{aligned}
$$


y por consiguiente,

$$
s_{1}\left((\alpha \otimes \beta)\left(\cup_{i=1}^{n} A_{i} \times B_{i}\right)=s\left(\sum_{i=1}^{n}\left(\beta\left(B_{i}\right) \cdot \alpha\left(A_{i}\right)(1)\right)\right)<\varepsilon .\right.
$$

Ahora bien, puesto que $B=\{y \in Y / q(y) \leq 1\} \subset \alpha(A)(\{1\})$, para cada colección finita $\left\{y_{1}, \ldots, y_{n}\right\} \subset B$ existen $A_{1}, \ldots, A_{n} \in A$ tales que $y_{i}=\alpha\left(A_{i}\right)(1)$ $(i=1, \ldots, n)$.

Se sigue de lo anterior que, si $\nu(B)<\frac{\delta}{K}(B \in \xi)$, entonces, para cada par tición medible y finita $\left\{B_{1}, \ldots, B_{n}\right\}$ de $B$ y cada colección $\left\{y_{1}, \ldots, y_{n}\right\} \subset$ $B, s\left(\sum_{i=1}^{n} \beta\left(B_{i}\right)\left(y_{i}\right)\right)<\varepsilon ; \mathrm{y}$, por tanto, $\|\beta\|_{s, q}(B) \leq \varepsilon$.

Así pues, $\|\beta\|_{s, Q} \ll \nu$, como queríamos demostrar.

\section{Bibliografía}

1. BARTLE, R. G., A general bilinear vector integral, Studia Math. 15 (1956), 337-352.

2. BRaVo DE LA PARRA, R., Tópicos en integración bilineal vectorial, Tesis Doctoral, Madrid, (1986).

3. BROOKS, J., On the existence of a control measure for strongly bounded vector measures, Bull. Amer. Math. Soc. 77 (1971), 999-1001.

4. DEBIEVE, C., Integration of vector valued functions with respect to vector valued measures, Rev. Roum. math. P. et Appl. 26 (1981), 943-957.

5. DIESTEL, J. Y UhL, J.J., Vector measures, Math. Surveys Amer. Math. Soc. Providence, R. I. 15 (1977).

6. Dobrakov, I., On integration in Banach spaces, I, Czech. Math. J. 20 (1970), 511-536.

7. FERNÁNDEZ Y FERNÁNDEZ-ARROYO, F.J., Producto de medidas valoradas en espacios localmente convexos, Tesis Doctoral, Madrid, (1987).

8. FERNÁNDEZ Y FERNÁNDEZ-ARROYO, F.J., On the product of operators valued measures, (En prensa).

9. FERNÁNDEZ Y FERNÁNDEZ-ARROYO, F.J., Integración en espacios localmente convexos, (En prensa).

10. KLUVÁNEK, I, Y KNOWLES, G., Vector Measures and Control Systems, "Nort-Holland," Amsterdam, (1975).

11. Rao Chivukula, R. y SASTRY, A. S., Product vector measures via Bartle integrais, J. Math. Anal. Appl. 96 (1983), 180-195.

12. RODRíGUEZ SALAZAR, S., Integración general en espacios localmente convexos, Tesis Doctoral, Madrid, (1985).

13. RODRÍGUEZ SALINAS, B., Integración de funciones con valores en un espacio localmente convexo, Rev. R. Acad. Ci. Madrid 73 (1979), 361-387. 
14. Sivasankara, S. A., Vector integrals and product of vector measures, Ph. D. Thesis, Univ. Microfilm, Inter. Michigan, (1983).

15. SWARTz, C., A generalization of a theorem of Duchon on products of vector measures, J. Math. Anal. Appl. 51 (1975), 621-628.

Departamento de Matemáticas Fundamentales

Facultad de Ciencias, U.N.E.D.

Ciudad Universitaria

28040-Madrid, SPAIN.

Rebut el 2 d'Octubre de 1987 